\title{
Primary total knee arthroplasty using constrained condylar knee design for severe deformity and stiffness of knee secondary to post-traumatic arthritis
}

\author{
Saroj Rai ${ }^{1}$, Xianzhe Liu ${ }^{1}$, Xiaobo Feng ${ }^{1}$, Bimal Rai ${ }^{2}$, Nira Tamang ${ }^{3}$, Jing Wang ${ }^{1}$, Shunan Ye ${ }^{1}$ and Shuhua Yang ${ }^{1 *}$
}

\begin{abstract}
Background: Key to a successful outcome of total knee arthroplasty (TKA) is to attain optimum alignment, adequate balance, and deformity correction. In primary TKA, this can be achieved efficiently by posterior stabilized (PS) design with or without the sub-periosteal release. However, certain circumstances such as post-traumatic arthritis are often associated with severe deformities with a significant bone defect, stiffness, and instability. Such deformities are extremely difficult to balance with soft tissue release only and require additionally constrained prostheses even in primary TKA. In such situation, constrained condylar knee (CCK) design is the ultimate choice. This study primarily aimed to report on clinical outcome, regain of function, and complication of patients who underwent primary CCK-TKA for severe deformity of the knee secondary to post-traumatic arthritis. The secondary aim was to find out the mid-term prostheses survival.
\end{abstract}

Methods: Between February 2007 and November 2013, 38 consecutive patients with post-traumatic arthritis of the knee received cemented primary CCK-TKA. Thirty-four patients (21 men and 13 women) who had a minimum of 3 years follow-up were included in this retrospective study. We used Knee Society Score (KSS), Hospital for Special Surgery (HSS) score, and roentgenographic evaluation form to assess the patients. Prostheses survival was assessed using Kaplan-Meier's survival analysis.

Results: Patients were followed up for an average duration of 6.47 years. KSS knee score improved from 44 points (23-68) pre-operatively to 91 points (76-100) post-operatively $[P<0.001]$. The average KSS functional score improved from 49 points (20-75) pre-operatively to 91 points $(65-100)$ post-operatively $[P<0.001]$. The average HSS score improved from 51 points (27-83) pre-operatively to 91 points (75-100) post-operatively $[P<0.001]$. Similarly, the average ROM improved from $68.09^{\circ} \pm 35.99^{\circ}\left(0^{\circ}-120^{\circ}\right)$ to $113.68^{\circ} \pm 8.90^{\circ}\left(100^{\circ}-130^{\circ}\right)$ post-operatively $[P<0.001]$. The average hip-knee-ankle (HKA) angle was $176.88^{\circ} \pm 14.48^{\circ}\left(135^{\circ}-199^{\circ}\right)$ pre-operatively and $180.24^{\circ} \pm 1.77^{\circ}\left(175^{\circ}-184^{\circ}\right)$ post-operatively. Radiolucencies were evident in 13 knees, mostly on the tibial side. Prostheses survival was $94.7 \%$ at a mean follow-up of 6.47 years.

Conclusion: Despite severe deformity, instability, and stiffness at a relatively young age, mid-term follow-up of primary CCK-TKA in post-traumatic arthritis provides satisfactory clinical and functional outcomes with 94. 7\% prostheses survival. However, it is not without complication.

Keywords: Total knee arthroplasty, Constrained condylar knee, Knee Society Score, Post-traumatic arthritis

\footnotetext{
*Correspondence: shuhua_yang@outlook.com

1 Department of Orthopaedics, Union Hospital, Tongji Medical College,

Huazhong University of Science and Technology, Wuhan 430022, China

Full list of author information is available at the end of the article
}

(c) The Author(s). 2018 Open Access This article is distributed under the terms of the Creative Commons Attribution 4.0 International License (http://creativecommons.org/licenses/by/4.0/), which permits unrestricted use, distribution, and reproduction in any medium, provided you give appropriate credit to the original author(s) and the source, provide a link to the Creative Commons license, and indicate if changes were made. The Creative Commons Public Domain Dedication waiver (http://creativecommons.org/publicdomain/zero/1.0/) applies to the data made available in this article, unless otherwise stated. 


\section{Background}

Intra-articular or peri-articular injuries are common in young and active individuals, which often lead to posttraumatic arthritis of the involved joint [1]. Prevalence of post-traumatic arthritis of the knee following injuries range from 21 to $44 \%$ [2] and is usually associated with severe bone loss and/or ligamentous injuries leading to a significant instability, stiffness, and severe deformity of the knee joint $[3,4]$.

Key to a successful outcome of total knee arthroplasty (TKA) is to attain optimum alignment, adequate balance, and deformity correction [5]. Generally, in primary TKA, this can be achieved efficiently by posterior stabilized (PS) design with or without the sub-periosteal release of ligaments. However, there are circumstances which require additionally constrained prostheses even in primary TKA such as severe bony defects and/or deformities and collateral ligament insufficiency that are extremely difficult to correct with soft tissue release only [5-7]. Soft tissue release in such situation somewhat jeopardizes the surrounding structures [8]. Additionally, in post-traumatic arthritis, there are always technical complexities owing to prior scars, the risk of infection, mal-alignment, knee stiffness, ligament insufficiency, and a significant bone loss. Such deformities warrant the use of constrained condylar knee (CCK) prosthesis with its variety of available stems and augments [3, 4, 9].

However, there are some disadvantages of CCK prostheses which include theoretical mechanical loosening due to load transfer to the respective bone ends via an intramedullary extension of the stems leading to early failure and a periprosthetic fracture $[8,10,11]$. Polyethylene insert wearing is another drawback of CCK [11]. Revision TKA following CCK prostheses is an extremely difficult procedure, as a need for stem removal increases significant morbidity and operating time [10].

Previous studies about the CCK-TKA focused mainly in two headings. First is the assessment of the effectiveness of CCK prostheses in different primary diagnoses including primary osteoarthritis, rheumatoid arthritis, post-traumatic arthritis, and Charcot arthropathy, leading to severe instability and/or deformity of the knee joint [7, $8,10-13]$. Second is the assessment of the outcome of primary TKA in post-traumatic arthritis using a variety of prostheses including cruciate retaining (CR), PS, CCK, or rotating hinge $(\mathrm{RH})[3,4,14-16]$. The majority of the studies reported improvement in the functional score post-operatively following CCK-TKA. However, till date, no study has focused on the use of CCK prostheses in primary TKAs following post-traumatic arthritis. This retrospective study primarily aimed to report on clinical outcome, regain of function, and complication of patients who underwent primary CCK-TKA for severe deformity of the knee secondary to post-traumatic arthritis with a minimum follow-up of 3 years. The secondary aim was to find out the mid-term prostheses survival.

\section{Methods \\ Patients}

Between February 2007 and November 2013, 38 consecutive patients with post-traumatic arthritis of the knee with severe deformity received cemented primary Zimmer $^{\bullet}$ NexGen $^{\bullet}$ Legacy $^{\oplus}$ Constrained Condylar Knee $\left(\mathrm{LCCK}^{\circ}\right)$ prostheses TKA. All the patients had unilateral CCK-TKA. Out of 38 patients, 1 patient died, 1 patient lost follow-up, 1 patient underwent implant removal secondary to prosthetic infection, and 1 patient had revision surgery for early prosthetic loosening, and they are excluded from the study though included in the prosthesis survival and complication rate. Eventually, 34 patients were available at the final follow-up and were included in the study. Detailed demographic characteristics of patients are well illustrated in Table 1. The average gap between trauma and CCK-TKA was $7.73 \pm$ 6.37 years $(1-26)$. There were 21 men and 13 women with an average age of $58 \pm 9.88$ years (33-73). Eighteen patients had left knee involvement, and 16 had right knee involvement. The average follow-up of patients was $6.47 \pm 1.99$ years (3-9.5). Out of 34 patients, $23(67.6 \%)$ patients had knee range of motion (ROM) of $90^{\circ}$ or less pre-operatively. Primary diagnosis included 20 (58.8\%) proximal tibia fractures, 9 (26.5\%) distal femur fractures, $4(11.8 \%)$ both tibia and femur fractures, and 1 (2.9\%) both tibia and femur with patella fractures. Thirty $(88.3 \%)$ patients had a history of surgical management of

Table 1 Demographics and characteristics of patients [Mean $\pm S D$, or $n(\%)]$

\begin{tabular}{lll}
\hline Demographic parameters $(N=34)$ & Mean \pm SD, or $n(\%)$ & Range \\
\hline Age (years) & $58 \pm 9.88$ & $33-73$ \\
Male/female & $21 / 13$ & \\
Right/left & $16 / 18$ & \\
BMl (kg/m²) & $24.86 \pm 1.27$ & $21.80-27.6$ \\
Trauma to TKA gap (years) & $7.73 \pm 6.37$ & $1-26$ \\
Prior surgery (yes/no) & $30 / 4$ & \\
Follow-up (years) & $6.47 \pm 1.99$ & \\
Primary diagnosis, $n$ (\%) & Proximal tibia fracture, & \\
& $20(58.8 \%)$ & \\
& Distal femur fracture, \\
& $9(26.5 \%)$ \\
& Tibia and femur fracture, \\
& $4(11.8 \%)$ \\
& Femur and patella fracture, \\
& $1(2.9 \%)$ \\
\hline
\end{tabular}

$N$ total sample, $n$ number of cases, BMI body mass index, TKA total knee arthroplasty, SD standard deviation 
the fracture, whereas $4(11.7 \%)$ patients received conservative management of the fracture.

Inclusion criteria were as follows: (1) patients who underwent a primary CCK-TKA for post-traumatic arthritis during the given period and had at least 3 years follow-up and (2) patients with previous knee surgery, such as prior osteotomies around the knee and/or nonarthroplasty implants like osteosynthesis plates or intramedullary nails. Exclusion criteria were as follows: (1) CCK-TKA for reasons other than post-traumatic arthritis; (2) patients underwent prior TKA or conversion of a unicompartmental knee arthroplasty (UKA); (3) patients who did not have pre-operative or post-operative imaging; and (4) arthroplasty associated with oncologic resection.

\section{Surgical procedure}

The decision to use CCK prosthesis was made by the experienced senior orthopedic surgeon (SHY) and his panel. Templating of pre-operative X-rays were performed on all knees. A standard medial parapatellar approach, anterior referencing, and measured resection technique were applied. Initially, tibial preparation was performed by a neutral tibial cut with $3^{\circ}$ of the posterior slope with an extramedullary guide. An intramedullary guide with a broach was applied for measurement of appropriate distal femoral cut parallel to the trans-epicondylar axis in $5^{\circ}-7^{\circ}$ valgus angle of anatomical axis. The decision to use CCK prosthesis was made pre-operatively in $60 \%$ of patients on the basis of severe deformity and instability where soft tissue release was not attempted. In $40 \%$ of patients, the decision was made intra-operatively when there was no adequate balance in flexion and/or extension even after an adequate sub-periosteal soft tissue release. A spacer block was used to assess extension and flexion gaps. Appropriately sized tibial and femoral provisional with a proper thickness spacer block was inserted. The operating surgeon (SHY) then evaluated stability by applying varusvalgus stress in extension, mid flexion, and at $90^{\circ}$ of flexion. The alignment was assessed using an extramedullary alignment rod. Finally, appropriately sized cemented tibial and femoral prostheses along with stems were implanted. Various bone graft or augments were used whenever there was a considerable bone defect. The lateral retinacular release was performed when intraoperative patella mal-tracking was evident. Routine patella resurfacing was not performed in our series; however, patella denervation with electrocautery was performed on all the knees.

\section{Rehabilitation}

Antibiotics and analgesics were routinely administered and continued for $48 \mathrm{~h}$ post-operatively. All patients were administrated with prophylactic anticoagulants. Rehabilitation started the next day with passive mobilization, followed by active rehabilitation. Early weight-bearing was usually encouraged. Early and late complications such as venous thromboembolism (VTE), hemorrhage, infection, dislocation, loosening, and periprosthetic fractures were continuously monitored in the ward and during the follow-ups. Standing anteroposterior (AP) and lateral view X-rays of the operated knee were taken in 3 days post-operatively and every follow-up visit for evaluation.

\section{Clinical and radiological evaluation}

The questionnaires were prepared according to the Knee Society Score (KSS) [17] and Hospital for Special Surgery (HSS) score [6]. KSS consisted of KSS knee score including ROM, and KSS functional score. Preoperative data such as demographic information and knee scores were collected retrospectively through the hospital database. Post-operative data were collected during follow-up visits. Demographic information such as age, gender, body mass index (BMI), side of the operated knee (right/left), and primary diagnoses were recorded. Patients were requested via a telephone call to visit the hospital for final follow-up even if they had left the follow-up in between.

Radiographs were evaluated for mechanical alignment (hip-knee-ankle (HKA) angle), anatomic alignment, and any radiolucent lines using the Knee Society Roentgenographic Evaluation form [18]. The last followup X-rays were compared with the immediate postoperative X-rays to look for the position of the implant, aseptic loosening, periprosthetic fracture, heterotrophic ossification (HO), and any other complications. The clinical and radiological evaluations were carried out by the orthopedic surgeon (XZL) who was not involved in the operation.

\section{Statistical analysis}

We used Statistical Package for Social Sciences (IBM SPSS Statistics 23) version 23 for statistical analysis. Categorical data were analyzed using chi-square test, and paired $t$ test was chosen for analysis of continuous parametric data, whereas the Mann-Whitney $U$ test was used to analyze nonparametric continuous data. KaplanMeier's survival analysis was used for prostheses survival. Results of categorical data were presented as frequencies and percentages, whereas results of continuous data were presented as the mean \pm standard deviation (SD) and range. Statistical differences were considered significant for $P$ values $<0.05$.

\section{Results}

Clinical results

Clinical scores are well illustrated in Table 2. The average KSS knee score improved from 44 points (23-68) pre- 
Table 2 Pre-operative versus post-operative outcomes of primary CCK-TKA in post-traumatic arthritis where all the parameters significantly improved post-operatively

\begin{tabular}{llll}
\hline Parameters & Pre-operative & Post-operative & $P$ value \\
$(N=34)$ & Mean \pm SD (Range) & Mean \pm SD (Range) & \\
\hline KSS Knee Score & $44.88 \pm 13.27(23-68)$ & $91.82 \pm 6.25(76-100)$ & $<0.001^{*}$ \\
KSS Functional Score & $49.12 \pm 14.16(20-75)$ & $91.03 \pm 9.75(65-100)$ & $<0.001^{*}$ \\
Hospital for Special & $51.00 \pm 13.49(27-83)$ & $91.68 \pm 7.20(75-100)$ & $<0.001^{*}$ \\
Surgery (HSS) score & & & \\
\hline
\end{tabular}

*Statistically significant differences were observed in all the parameters

$N$ sample size, SD standard deviation, KSS Knee Society Score

operatively to 91 points $(76-100)$ at the final follow-up $[P$ $<0.001]$. Out of 34 patients, $32(94.1 \%)$ patients were rated as excellent and $2(5.9 \%)$ patients were rated as good. The average KSS functional score improved from 49 points (20-75) pre-operatively to 91 points $(65-100)$ at the final follow-up $[P<0.001]$. The average HSS score improved from 51 points (27-83) pre-operatively to 91 points (75100) at the final follow-up $[P<0.001]$.

Similarly, the average ROM improved from $68.09^{\circ} \pm$ $35.99^{\circ}\left(0^{\circ}-120^{\circ}\right)$ to $113.68^{\circ} \pm 8.90^{\circ}\left(100^{\circ}-130^{\circ}\right)$ at the final follow-up $[P<0.001]$ (Fig. 1). Pre-operatively, 23 $(67.6 \%)$ patients had average flexion contracture of $17^{\circ}$ $\left(5^{\circ}-45^{\circ}\right)$; post-operatively, $1(2.9 \%)$ patient had flexion contracture of $5^{\circ}$. Similarly, $2(5.9 \%)$ patients had preoperative extension lag of $5^{\circ}$, but none of the patients had extension lag post-operatively.

\section{Radiographic results}

The pre-operative radiographic evaluation showed an average varus deformity of $12^{\circ}\left(0^{\circ}-30^{\circ}\right)$ in $10(29.4 \%)$ patients, and an average valgus deformity of $15^{\circ}$ (7.5-25) in $17(50 \%)$ patients in weight-bearing AP radiographs, whereas $7(20.6 \%)$ patients had a normal valgus angle of $3^{\circ}$ to $7^{\circ}$. There were no deformities at the final followup. The average HKA angle was $176.88^{\circ} \pm 14.48^{\circ}\left(135^{\circ}-\right.$ $\left.199^{\circ}\right)$ pre-operatively and $180.24^{\circ} \pm 1.77^{\circ}\left(175^{\circ}-184^{\circ}\right)$ at the final follow-up (Figs. 2 and 3). Radiolucencies were evident in 13 knees, 10 were on the tibial side and 3 on both sides. However, all these radiolucencies were less than $1 \mathrm{~mm}$ in size and were evident also in immediate post-operative radiographs.

\section{Prosthesis survival}

We used endpoint as a revision for any reason for all 38 cases. Prostheses survival was $94.7 \%$ (95\% confidence interval (CI) 82.3 99.4\%) at an average follow-up of 6.47 years.

\section{Complication}

Complications are well illustrated in Table 3. Two patients, a patient with an implant loosening in 1 month and a patient with prosthetic infection in 2.5 years postoperatively, underwent prostheses removal followed by revision procedures. A patient who suffered nondisplaced periprosthetic fracture around the tibial stem which was managed conservatively reported a good outcome. Three patients had symptomatic deep vein thrombosis (DVT); however, all of them recovered well after early diagnosis and essential management. Common peroneal nerve palsy occurred in one patient, whose motor symptoms recovered after 6 months post-

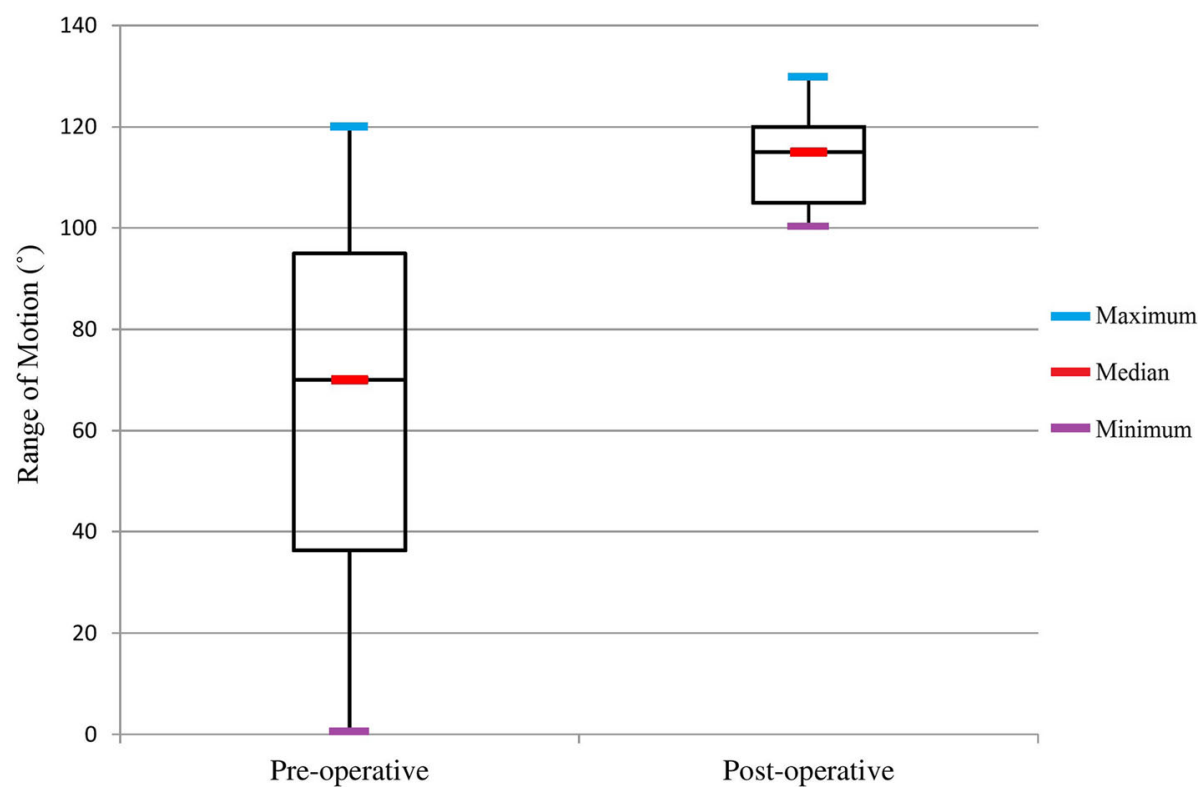

Fig. 1 The pre-operative and post-operative range of motion (ROM) of patients 

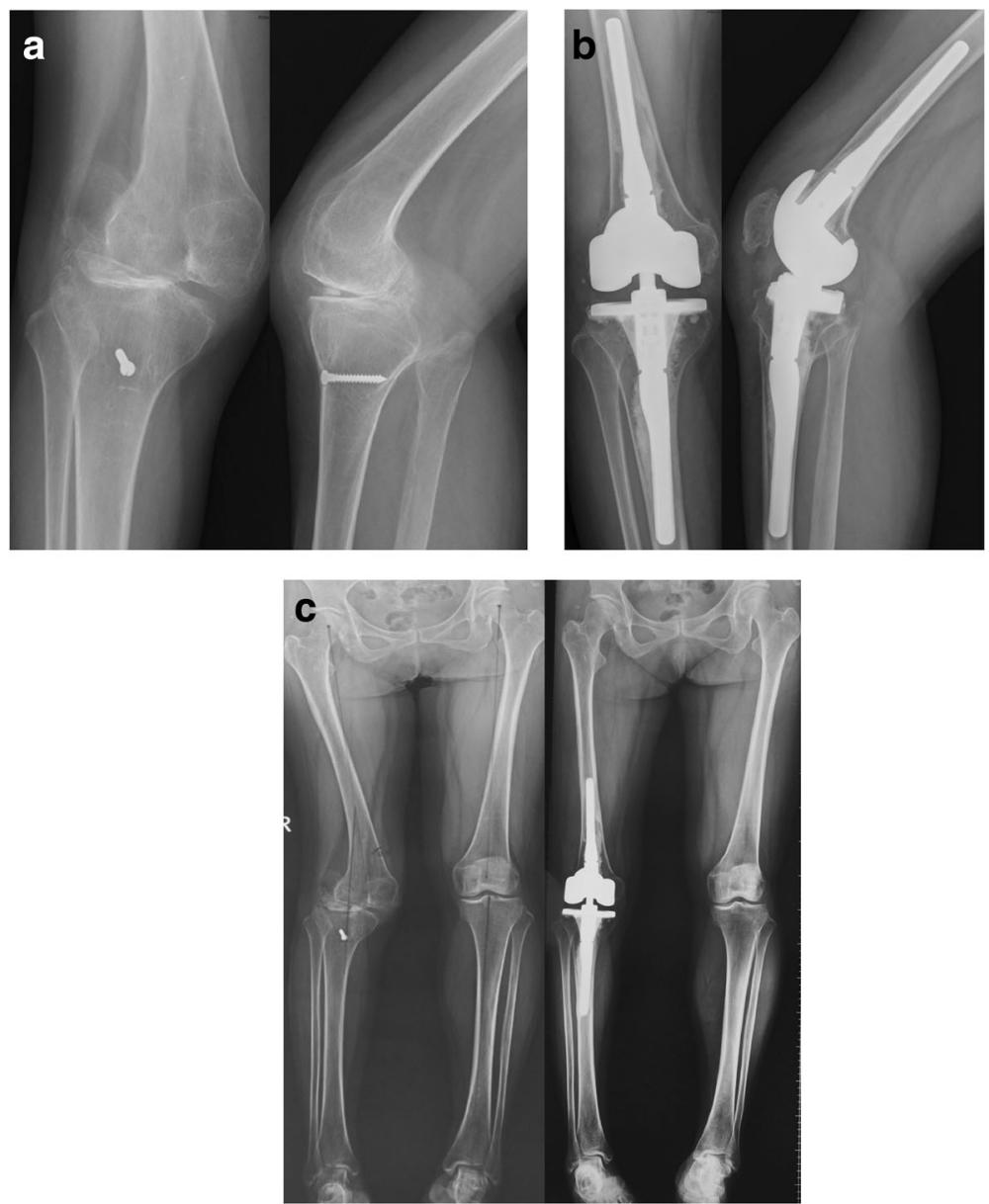

Fig. 2 Implantation of CCK prosthesis in a 62-year-old female with post-traumatic arthritis with valgus deformity and significant instability of the right knee. a Pre-operative anteroposterior (AP) and lateral radiographs; she had undergone some type of procedure on her index knee. b Post-operative radiograph shows $3^{\circ}$ of valgus and no radiolucent lines. c Comparison of pre-operative and post-operative long leg radiographs

operatively. Heterotropic ossification (HO) occurred in one, and asymptomatic patella baja occurred in one patient.

\section{Discussion}

The most important finding of our study was that the primary CCK-TKA successfully corrected the severe deformity with significant bone defects, stiffness, and instability of the knee joint secondary to post-traumatic arthritis with prostheses survival rate of $94.7 \%$ restoring excellent clinical outcome and regain of function. However, post-operative complication rate was as high as $24 \%$.

Previously, Lachiewicz and Soileau [13] studied the results of second-generation primary CCK-TKA in 27 knees; there was a significant improvement in all the parameters including KSS knee score, KSS functional score, and HSS score post-operatively at the final followup. Using KSS knee score, 12 (44\%) knees rated as excellent, $14(52 \%)$ as good, and $1(4 \%)$ as fair. Ye et al. [19] compared the outcome of CCK-TKA in complex primary and revision surgery and reported no significant difference between two groups at a mean follow-up of 5.5 years post-operatively. However, they found a significant improvement in the clinical scores with 92\% knees as having good to excellent results in the primary CCK group [19]. Moreover, Lizaur-Utrilla et al. [20] provided a similar opinion with a significant improvement postoperatively yet found no significant differences in the post-operative outcomes of the patients who underwent primary TKA for post-traumatic arthritis versus primary osteoarthritis. However, Lunebourg et al. [15] reported that regardless of having a significant difference in the post-operative scores of primary TKA, the postoperative scores of TKA following post-traumatic arthritis was lower than that of the primary osteoarthritis group. This difference was possibly due to the poor preoperative status of patients, not because of the intrinsic success of the procedure [15]. In our study, mid-term results of cemented primary CCK-TKA in posttraumatic arthritis was evaluated using KSS knee score, KSS functional score, and HSS score. Regardless of 

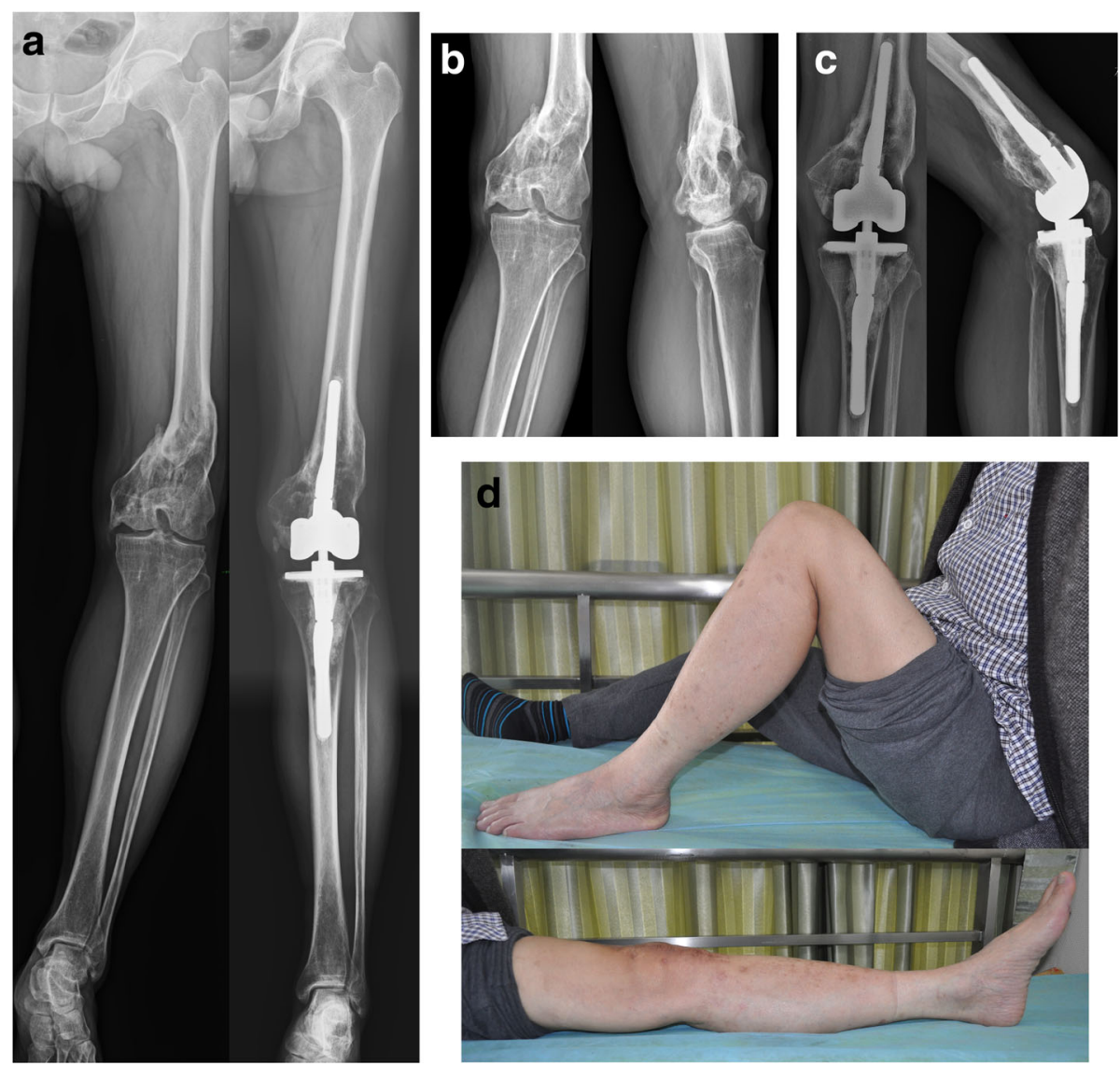

Fig. 3 CCK prosthesis in a 58-year-old male patient with the mal-united distal femur and post-traumatic arthritis of the left knee. The patient was having knee stiffness and patella baja for 20 years due to post-traumatic scar. a Comparison of pre-operative and post-operative long leg radiographs in anteroposterior (AP) view. b, c Pre-operative and post-operative radiographs respectively. $\mathbf{d}$ Clinical photographs showing a post-operative range of motion (ROM)

having severe deformity, stiffness, and instability preoperatively, 94\% patients had excellent post-operative scores at the last follow-up, which was comparable with reports mentioned above.

Another important finding of our study was a significant improvement in the post-operative ROM. Although our patients had poor preoperative ROM with an average of

Table 3 Complications of primary CCK-TKA in post-traumatic arthritis (38 knees)

\begin{tabular}{llc}
\hline Complications & Knees, $n(\%)$ & Revision $n(\%)$ \\
\hline Deep venous thrombosis & $3(7.89)$ & \\
Infection & $1(2.63)$ & $1(2.63)$ \\
Periprosthetic fracture & $1(2.63)$ & $1(2.63)$ \\
Prosthetic loosening & $1(2.63)$ & \\
Common peroneal nerve injury & $1(2.63)$ & \\
Heterotropic ossification & $1(2.63)$ & $2(5.26)$ \\
Patella baja & $1(2.63)$ & \\
Overall & $9(23.68)$ & \\
\hline
\end{tabular}

$n$ number of cases $68^{\circ}$, it improved significantly with an average ROM of $113^{\circ}$ at the final follow-up. Our finding was consistent with previous reports as the majority of studies reported post-operative ROM of CCK prostheses ranging from $89.4^{\circ}$ to $117^{\circ}[8,11,21-24]$. Maynard et al. [24] reported post-operative ROM to be dependent on pre-operative ROM. In our study, even though 23 (67.6\%) knees had a pre-operative ROM of $90^{\circ}$ or less, post-operatively the ROM improved satisfactorily with all having ROM of $100^{\circ}$ or more. Considering this report, we can conclude that post-operative ROM depends not only on pre-operative $\mathrm{ROM}$, but also on adequate intra-operative balancing, and appropriate rehabilitation.

Despite having a significant improvement in clinical and functional outcome following TKA in posttraumatic arthritis, the complication rate is often high (Table 4) [2]. Complications include infection, wound complications, intra-operative rupture of the patellar tendon, stiffness, and mechanical loosening [2]. Various factors contribute to the complications after TKA in post-traumatic arthritis such as prior fracture surgeries, 
Table 4 A literature review of complications rate of primary TKA after post-traumatic arthritis

\begin{tabular}{|c|c|c|c|c|c|c|c|c|}
\hline \multirow[b]{2}{*}{ Parameters } & \multicolumn{8}{|c|}{ References } \\
\hline & $\begin{array}{l}\text { Massin } \\
\text { et al. [30] }\end{array}$ & $\begin{array}{l}\text { Abdel } \\
\text { et al. [27] }\end{array}$ & $\begin{array}{l}\text { Lonner } \\
\text { et al. [29] }\end{array}$ & $\begin{array}{l}\text { Papadopoulos } \\
\text { et al. [28] }\end{array}$ & $\begin{array}{l}\text { Parratte } \\
\text { et al. [16] }\end{array}$ & $\begin{array}{l}\text { Lunebourg } \\
\text { et al. [15] }\end{array}$ & $\begin{array}{l}\text { Lizaur-utrilla } \\
\text { et al. [20] }\end{array}$ & $\begin{array}{l}\text { Weiss } \\
\text { et al. [3] }\end{array}$ \\
\hline Sample size $(N)$ & 40 & 62 & 31 & 48 & 74 & 33 & 29 & 110 \\
\hline Male/female & $21 / 19$ & $22 / 40$ & 15/15(1-BL) & 10/37(1-BL) & $34 / 40$ & $18 / 15$ & $10 / 19$ & $32 / 77(1-B L)$ \\
\hline Age (years) & 59 & 63 & 60 & 65 & 63 & 69 & 57 & 64 \\
\hline Follow-up (years) & 5 & 15 & 4 & 6 & 4 & 11 & 7 & 5.5 \\
\hline \multicolumn{9}{|l|}{ Complications n (\%) } \\
\hline Venous thrombosis & & $1(1.6)$ & $2(6.4)$ & & & & & $6(5.5)$ \\
\hline Aseptic failure & & & $8(25.8)$ & & $1(1.7)$ & $1(3.0)$ & & $4(3.6)$ \\
\hline Infection & $2(5.0)$ & $5(8.0)$ & $3(9.6)$ & $3(6.2)$ & $4(6.8)$ & $2(6.1)$ & $1(3.4)$ & $5(4.5)$ \\
\hline Stiffness & & $6(9.6)$ & & & $6(10.2)$ & $2(6.1)$ & $1(3.4)$ & $8(7.3)$ \\
\hline Patellar tendon rupture & $3(7.5)$ & & $1(3.2)$ & $1(2.1)$ & $3(5.1)$ & & $1(3.4)$ & \\
\hline Ligament rupture & & $1(1.6)$ & & & & & & $1(0.9)$ \\
\hline Patella subluxation & & $2(3.2)$ & $2(6.4)$ & $1(2.1)$ & & & & \\
\hline Patella clunk syndrome & & & & & $1(1.7)$ & $1(3.0)$ & & $1(0.9)$ \\
\hline Skin necrosis and wound dehiscence & $2(5)$ & $3(4.8)$ & $2(6.4)$ & $2(4.2)$ & & & $1(3.4)$ & $5(4.5)$ \\
\hline Peroneal nerve injury & & & & & $1(1.7)$ & & & \\
\hline Popliteal artery injury & $1(2.5)$ & & & & $1(1.7)$ & & & \\
\hline Periprosthetic fractures & $1(2.5)$ & $1(1.6)$ & & & & & & \\
\hline Pseudoarthrosis & $1(2.5)$ & & & & & & & \\
\hline Instability & & & & & $1(1.7)$ & & & $2(1.8)$ \\
\hline Heterotropic ossification & & & & & & $1(3.0)$ & & \\
\hline Hematoma & & $1(1.6)$ & & $2(4.2)$ & $2(3.4)$ & & & \\
\hline Reflex sympathetic dystrophy & & $1(1.6)$ & & & & & & $1(0.9)$ \\
\hline Overall & $11(27.5)$ & 21(33.8) & $18(58)$ & $9(18.7)$ & $20(33.9)$ & $7(21.2)$ & $4(13.7)$ & $33(30.0)$ \\
\hline
\end{tabular}

$N$ sample size, $n$ number of cases, $B L$ bilateral

scarring of soft tissue, severe joint deformity, and malpositioning of the implants. Piedade et al. [25] reported that previous surgery around the knee predisposes to higher-rate post-operative complications after TKA. Prosthetic infection is regarded as most frequent and a dreadful complication following TKA and is the primary reason for re-operation [11]. Jamsen et al. [26] reported that prior fracture or use of constrained prosthesis was a risk factor for infection. In our study, overall complications occurred in 9 (23.7\%) knees out of 38 patients as mentioned above and 2 (5.26\%) patients (2 knees) needed revision TKA. Previous studies reported that the overall infection rate in primary TKA secondary to posttraumatic arthritis was between 3.4 and $9.6 \%[3,15,16$, 20, 27-29]. Regardless of the use of CCK in a relatively vulnerable group of patients, prosthetic infection occurred in only one $(2.63 \%)$ patient in 2.5 years following primary TKA that needed prosthetic removal. A relatively low infection rate might be due to strict intraoperative aseptic precautions along with the routine use of prophylactic antibiotics. Another revision occurred in a male patient with distal femoral fracture with prior surgeries followed by knee stiffness and considerable deformity for more than 20 years. Primarily, we used LCCK prosthesis with stem extension in femoral component and without stem extension in the tibial component; unfortunately, aseptic tibial component prosthetic loosening occurred at 1 month following TKA necessitating a revision surgery with a long stem. We regarded the reason for loosening to be wrong prosthesis choice leading to mal-positioning $[3,16]$. In this study, we found the survival rate prostheses to be $94.7 \%$ (95\% CI 82.3 99.4\%) and was fairly consistent with the previous literature of long-term follow-up when the revision of any cause was set as an endpoint $[11,21]$.

Our study has several limitations. Our study has all the biases related to a retrospective cohort study with a relatively small sample size. Selection bias is another limitation as the choice of the prosthesis was at the decision of the chief operating surgeon. Lastly, the results presented here are from a single hospital and may reflect regional and institutional bias. 


\section{Conclusion}

This study demonstrates that despite having a severe deformity and stiffness of the knee joint at a relatively young age, mid-term follow-up of primary CCK-TKA in post-traumatic arthritis provides satisfactory clinical and functional outcomes with $94.7 \%$ prostheses survival. Considering this report, we can conclude that CCK-TKA is effective and a good option for the treatment of posttraumatic arthritis of the knee. However, it is not without complication. Long-term follow-up is warranted to obtain an ultimate conclusion.

\section{Abbreviations}

AP: Anteroposterior; BMI: Body mass index; CCK: Constrained condylar knee; CR: Cruciate retaining; HKA: Hip-knee-ankle; HO: Heterotrophic ossification: HSS: Hospital for Special Surgery score; KSS: Knee Society Score; PS: Posterior stabilized; RH: Rotating hinge; ROM: Range of motion; TKA: Total knee arthroplasty; UKA: Unicompartmental arthroplasty; VTE: Venous thromboembolism

\section{Acknowledgements}

Not applicable.

\section{Funding}

National Natural Science Foundation of China (Grant Number: 81772344).

\section{Availability of data and materials}

The data sets supporting the conclusion of this article are included in the manuscript. Upon request, raw data can be provided by the corresponding author.

\section{Authors' contributions}

SR designed and wrote the manuscript. XZL collected the data. BR, NT, and SR analyzed the data. XBF, JW, and SNY reviewed the literature, and SHY performed all the surgeries and supervised this study. All the authors carefully read and approved the final manuscript.

\section{Ethics approval and consent to participate}

The study was approved by the Institutional Review Board (IRB) of Tongji Medical College. Each author certifies that all the investigations were conducted in conformity with ethical principles. All the patients provided written informed consent.

\section{Consent for publication}

We obtained a written informed consent from the patient for publication of images.

\section{Competing interests}

The authors declare that they have no competing interests.

\section{Publisher's Note}

Springer Nature remains neutral with regard to jurisdictional claims in published maps and institutional affiliations.

\footnotetext{
Author details

${ }^{1}$ Department of Orthopaedics, Union Hospital, Tongji Medical College, Huazhong University of Science and Technology, Wuhan 430022, China. 2Department of Radiology, Tongji Hospital, Tongji Medical College, Huazhong University of Science and Technology, Wuhan 430030, China. ${ }^{3}$ School of Nursing, Tongji Medical College, Huazhong University of Science and Technology, Wuhan 430030, China.
}

Received: 8 April 2017 Accepted: 7 March 2018

Published online: 02 April 2018

\section{References}

1. Pickering RD. Posttraumatic arthritis. Can Fam Physician. 1984;30:1511-3.
2. Saleh H, Yu S, Vigdorchik J, Schwarzkopf R. Total knee arthroplasty for treatment of post-traumatic arthritis: systematic review. World J Orthop. 2016;7(9):584-91.

3. Weiss NG, Parvizi J, Hanssen AD, Trousdale RT, Lewallen DG. Total knee arthroplasty in post-traumatic arthrosis of the knee. J Arthroplast. 2003;18(3 Suppl 1):23-6.

4. Bala A, Penrose CT, Seyler TM, Mather RC 3rd, Wellman SS, Bolognesi MP. Outcomes after total knee arthroplasty for post-traumatic arthritis. Knee. 2015;22(6):630-9.

5. Sculco TP. The role of constraint in total knee arthoplasty. J Arthroplast. 2006;21(4 Suppl 1):54-6.

6. Insall JN, Ranawat CS, Aglietti P, Shine J. A comparison of four models of total knee-replacement prostheses. J Bone Joint Surg Am. 1976;58(6): 754-65.

7. Donaldson WF 3rd, Sculco TP, Insall JN, Ranawat CS. Total condylar III knee prosthesis. Long-term follow-up study. Clin Orthop Relat Res. 1988;226:21-8.

8. Easley ME, Insall JN, Scuderi GR, Bullek DD. Primary constrained condylar knee arthroplasty for the arthritic valgus knee. Clin Orthop Relat Res. 2000; 380:58-64.

9. Mozella Ade P, Olivero RR, Alexandre H, Cobra AB. Use of a trabecular metal cone made of tantalum, to treat bone defects during revision knee arthroplasty. Rev Bras Ortop. 2014;49(3):245-51.

10. Anderson JA, Baldini A, MacDonald JH, Pellicci PM, Sculco TP. Primary constrained condylar knee arthroplasty without stem extensions for the valgus knee. Clin Orthop Relat Res. 2006;442:199-203.

11. Cholewinski P, Putman S, Vasseur L, Migaud H, Duhamel A, Behal H, Pasquier G. Long-term outcomes of primary constrained condylar knee arthroplasty. Orthop Traumatol Surg Res. 2015;101(4):449-54.

12. Hartford JM, Goodman SB, Schurman DJ, Knoblick G. Complex primary and revision total knee arthroplasty using the condylar constrained prosthesis: an average 5-year follow-up. J Arthroplast. 1998;13(4):380-7.

13. Lachiewicz PF, Soileau ES. Results of a second-generation constrained condylar prosthesis in primary total knee arthroplasty. J Arthroplast. 2011; 26(8):1228-31.

14. Weiss NG, Parvizi J, Trousdale RT, Bryce RD, Lewallen DG. Total knee arthroplasty in patients with a prior fracture of the tibial plateau. J Bone Joint Surg Am. 2003;85-A(2):218-21.

15. Lunebourg A, Parratte S, Gay A, Ollivier M, Garcia-Parra K, Argenson JN. Lower function, quality of life, and survival rate after total knee arthroplasty for posttraumatic arthritis than for primary arthritis. Acta Orthop. 2015;86(2): 189-94.

16. Parratte $S$, Boyer P, Piriou P, Argenson JN, Deschamps G, Massin P, Sfhg. Total knee replacement following intra-articular malunion. Orthop Traumatol Surg Res. 2011;97(6 Suppl):S118-23.

17. Insall JN, Dorr LD, Scott RD, Scott WN. Rationale of the knee society clinical rating system. Clin Orthop Relat Res. 1989;248:13-4.

18. Ewald FC. The knee society total knee arthroplasty roentgenographic evaluation and scoring system. Clin Orthop Relat Res. 1989;248:9-12.

19. Ye CY, Xue DT, Jiang S, He RX. Results of a second-generation constrained condylar prosthesis in complex primary and revision total knee arthroplasty: a mean 5.5-year follow-up. Chin Med J. 2016;129(11):1334-9.

20. Lizaur-Utrilla A, Collados-Maestre I, Miralles-Munoz FA, Lopez-Prats FA. Total knee arthroplasty for osteoarthritis secondary to fracture of the tibial plateau. A prospective matched cohort study. J Arthroplast. 2015;30(8): 1328-32.

21. Lachiewicz PF, Soileau ES. Ten-year survival and clinical results of constrained components in primary total knee arthroplasty. J Arthroplast. 2006;21(6):803-8.

22. Hohl WM, Crawfurd E, Zelicof SB, Ewald FC. The total condylar III prosthesis in complex knee reconstruction. Clin Orthop Relat Res. 1991;273:91-7.

23. Chotivichit AL, Cracchiolo A 3rd, Chow GH, Dorey F. Total knee arthroplasty using the total condylar III knee prosthesis. J Arthroplast. 1991;6(4):341-50.

24. Maynard LM, Sauber TJ, Kostopoulos VK, Lavigne GS, Sewecke JJ, Sotereanos NG. Survival of primary condylar-constrained total knee arthroplasty at a minimum of 7 years. J Arthroplast. 2014;29(6):1197-201.

25. Piedade SR, Pinaroli A, Servien E, Neyret P. TKA outcomes after prior bone and soft tissue knee surgery. Knee Surg Sports Traumatol Arthrosc. 2013, 21(12):2737-43.

26. Jamsen E, Huhtala H, Puolakka T, Moilanen T. Risk factors for infection after knee arthroplasty. A register-based analysis of 43,149 cases. J Bone Joint Surg Am. 2009;91(1):38-47. 
27. Abdel MP, von Roth P, Cross WW, Berry DJ, Trousdale RT, Lewallen DG. Total knee arthroplasty in patients with a prior tibial plateau fracture: a long-term report at 15 years. J Arthroplast. 2015;30(12):2170-2.

28. Papadopoulos EC, Parvizi J, Lai CH, Lewallen DG. Total knee arthroplasty following prior distal femoral fracture. Knee. 2002;9(4):267-74.

29. Lonner JH, Pedlow FX, Siliski JM. Total knee arthroplasty for post-traumatic arthrosis. J Arthroplast. 1999;14(8):969-75.

30. Massin P, Bonnin M, Paratte S, Vargas R, Piriou P, Deschamps G, French Hip Knee $S$. Total knee replacement in post-traumatic arthritic knees with limitation of flexion. Orthop Traumatol Surg Res. 2011;97(1):28-33.

Submit your next manuscript to BioMed Central and we will help you at every step:

- We accept pre-submission inquiries

- Our selector tool helps you to find the most relevant journal

- We provide round the clock customer support

- Convenient online submission

- Thorough peer review

- Inclusion in PubMed and all major indexing services

- Maximum visibility for your research

Submit your manuscript at www.biomedcentral.com/submit
Biomed Central 